\title{
Desmopressin Acetate
}

National Cancer Institute

\section{Source}

National Cancer Institute. Desmopressin Acetate. NCI Thesaurus. Code C47475.

An analogue of the hormone vasopressin with antidiuretic and antihemorrhagic

properties. Desmopressin acetate has selective affinity for the V2 receptor and acts on the distal kidney tubule by increasing the cellular permeability thereby stimulating water reabsorption. This antidiuretic agent is used in the treatment of central diabetes insipidus. An unrelated action of desmopressin acetate is to increases circulating factor VIII and is used in patients with haemophilia and von Willebrand's disease. 